\section{Large-eddy simulations of Richtmyer-Meshkov instability in a converging geometry}

\section{Lombardini ${ }^{1}$ and R. Deiterding ${ }^{2}$}

${ }^{1}$ Graduate Aeronautical Laboratories, California Institute

of Technology, Pasadena, California 91125, USA

${ }^{2}$ Computer Science and Mathematics Division, Oak Ridge

National Laboratory, Oak Ridge, Tennessee 37831-6367, USA

(Received 1 September 2010; published online 30 September 2010)

[doi:10.1063/1.3491373]

The Richtmyer-Meshkov instability (RMI) refers to the baroclinic generation of vorticity at a perturbed density interface when impacted by a shock wave. It is often thought of as the impulsive limit of the Rayleigh-Taylor instability. While the RMI has been widely covered in planar geometries (see, for example, Ref. 1), the present simulations investigate the mixing of materials resulting from the interaction of an imploding cylindrical shock wave with a concentric interface, perturbed in both axial and azimuthal directions, which separates outside air from $\mathrm{SF}_{6}$ (initially 5 times denser) confined in a $90^{\circ}$ wedge. Two incident shocks of Mach numbers $M_{i}=1.3$ and 2.0 at initial impact are tested. These canonical simulations support recent work on understanding the compressible turbulent mixing in converging geometries relevant to both inertial confinement fusion and core-collapse supernova dynamics.

Initial irregularities in the density interface form the misalignment between density and pressure gradients required to initiate a first RMI. A second RMI occurs after the initial shock has converged down the wedge, reflected off the axis and reshocks the distorted interface. Reshock interactions of decreasing intensity follow successively. Due to the converging geometry, the accelerated or decelerated motion of the interface also generates Rayleigh-Taylor instabilities. Secondary Kelvin-Helmholtz instabilities develop along the sides of the interpenetrating fingering structures.

The energetic reshock produces a large dynamical range of turbulent scales, requiring the utilization of large-eddy simulation (LES). We employed the stretched-vortex subgrid-scale model of turbulent and scalar transport based on an explicit structural modeling of small-scale dynamics. ${ }^{2}$ The imploding nature of the flow is particularly suitable for the use of adaptive mesh refinement (AMR) provided by the parallel block-structured AMR framework AMROC. ${ }^{3}$ The Favre-filtered Navier-Stokes equations are solved on each Cartesian uniform subgrid of the mesh hierarchy. A weighted, essentially nonoscillatory scheme is used to capture discontinuities but reverts to a low-numerical dissipation, explicit, tuned center-difference stencil in the smooth or

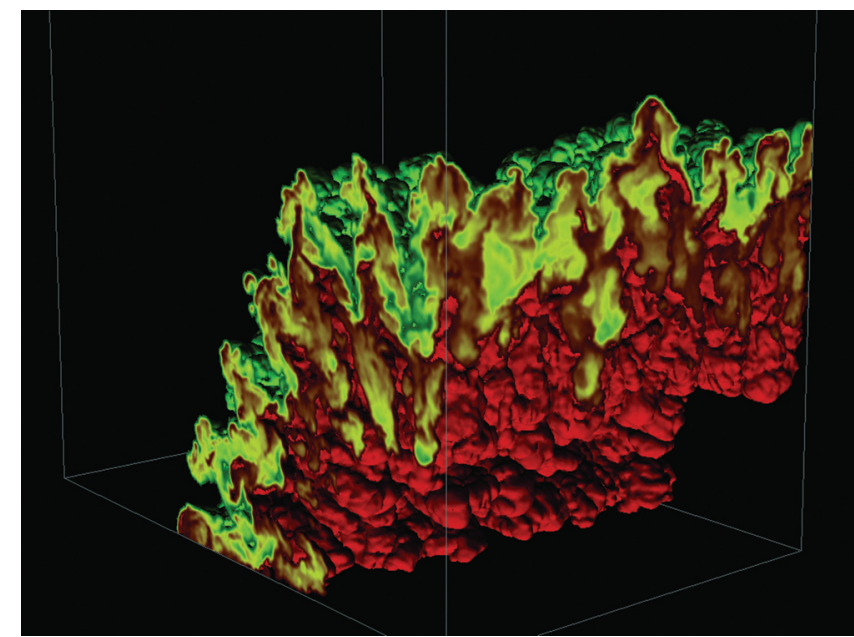

FIG. 1. (Color) (enhanced online)

[URL: http://dx.doi.org/10.1063/1.3491373.1].

turbulent flow regions, optimal for the functioning of our explicit LES (see Ref. 4 for more details).

The computational domain is discretized with $95 \times 95$ $\times 64$ cubic cells on the base grid (64 points in the periodic axial direction), with three additional levels of refinement based on the local density gradient, reducing the computational expenses compared to the equivalent finest unigrid $760 \times 760 \times 512$ problem. The interpenetration of the two fluids after the reshock is clearly discernible in Fig. 1. The mixing is visualized by using isosurfaces of the heavy-fluid mass fraction $\psi$. The mixture is colored red as $\psi=0.90$, yellow as $\psi=0.5$, and green as $\psi=0.1$. To illustrate the multiscale nature of the turbulent mixing, we observe that the initial perturbation wavelength is 100 times larger than the finest grid size, which is itself about 100 times larger than the estimated smallest physical scale in the flow-the Kolmogorov scale-where the viscous dissipation of energy occurs. Incident shocks of higher Mach number (e.g., $M_{i}=2.0$ ) focus the flow further towards the axis, leading to a stronger turbulent mixing.

This work was supported by the ASC Program under Subcontract No. B341492 of DoE Contract No. W-7405ENG-48.

\footnotetext{
${ }^{1}$ M. Vetter and B. Sturtevant, "Experiments on the Richtmyer-Meshkov instability of an air/SF 6 interface," Shock Waves 4, 247 (1995).

${ }^{2}$ A. Misra and D. I. Pullin, "A vortex-based subgrid stress model for largeeddy simulation," Phys. Fluids 9, 2443 (1997).

${ }^{3}$ R. Dieterding, R. Radovitzki, S. Mauch, F. Cirak, D. J. Hill, C. Pantano, J. C. Cummings, and D. I. Meiron, "The Virtual Test Facility: A virtual shock physics facility for simulating the dynamic response of materials," available at http://www.cacr.caltech.edu/asc.

${ }^{4}$ M. Lombardini, R. Deiterding, and D. I. Pullin, in Quality and Reliability of Large-Eddy Simulations, Proceedings of QLES 2007 International Symposium, Leuven, Belgium, edited by J. Meyers, B. Geurts, and P. Sagaut (Springer, Dordrecht, 2008), Vol. 12, pp. 283-294.
} 